\title{
How to improve patient experience of compassion through nursing: Development of an on-job mentorship programme in a tertiary care hospital, Pakistan
}

\author{
Alma Arshad \\ Aga Khan University \\ Naureen Lalani \\ Aga Khan University Hospital \\ Noureen Sultan \\ Aga Khan University Hospital \\ Aly Zubairi \\ Charter for Compassion \\ Ayesha Hussain \\ Caravan Life Trust \\ Babar S. Hasan \\ Aga Khan University \\ Muneera A. Rasheed ( $\nabla$ muneera.rasheed@aku.edu ) \\ Aga Khan University https://orcid.org/0000-0002-9064-0102
}

Research article

Keywords: compassion, nurses, on-job mentorship, Pakistan, patient experience, Theory of Change

Posted Date: December 10th, 2020

DOI: https://doi.org/10.21203/rs.3.rs-122995/v1

License: (c) (i) This work is licensed under a Creative Commons Attribution 4.0 International License.

Read Full License 


\section{Abstract \\ Background}

Evidence suggests improvement in nursing staff satisfaction, competence, and retention after implementation of evidence-based mentorship programmes. When guided by a framework of compassion, mentoring as a caring action can not only build healthy, transformative relationships and energize the workplace environments but a similar behavior is reciprocated to patients which subsequently can drive patient experience of care. However, examples of on-job mentorship programs for nurses in low- and middle-income countries (LMIC) are limited owing to human and financial constraints.

\section{Objective}

The current study describes the development of a feasible an on-job nursing mentorship program using a compassionate framework aimed at improving nurses' experience and thus enhancing patient experience from a tertiary care hospital in Pakistan.

\section{Methods}

The intervention package was built by a team composed of service and nursing leadership, director patient experience of care and a compassion specialist using a theory of change model. The package followed a series of steps that were based on the principles of implementation science, a) identification of a framework, b) creating a working group and c) multiple meetings to frame the model followed by implementing the preconditions for roll-out of the programme.

\section{Results}

The intermediate outcome was to have nurses demonstrate skills of compassion with the eventual outcome of improving the patient's experience of compassion. The pre-conditions were identified as: recruitment of staff with appropriate skills for pediatric care, precision of compassionate experience definition, creation of a compassionate culture and development of competent head nurses as supervisor. In order to ensure the pre-conditions, various interventions were planned and some were implemented through the course of designing. These involved, creation of space to talk about compassionate skills with staff, provide trainings and on-job coaching, revising career ladder of supervisors, inclusion of pediatric compassion specific nursing education service orientation program, recognition of efforts and promotion of compassionate values in numerous communication platforms, arrangement of training workshops, creation of checklists and development of dashboards.

\section{Conclusion}


The approach helped to delineate feasible pathways for an on-job compassionate mentorship programme through strengthening routine supervision leading to improved patient experience of care in the pediatric service.

\section{Background}

Patient-centeredness defined as 'providing care that is respectful of and responsive to, individual patient preferences, needs and values, and ensuring that patient values guide all clinical decisions' by the Institute of Medicine has lately been acknowledged as an essence to best quality services (1). The implementation of patient-centeredness also requires direct interaction and a strong relation with the patient which mostly happens through nurses. Owing to the characteristic frequency of nurse-patient interactions, they naturally emerge as a gateway for improving patient experience. The nurse-patient relation is strong to such an extent that the practice nurses become a central point in shaping and improving patients' experience (2). Hence, apart from possessing the knowledge and technical skills, demonstrating compassionate behaviors is essential to tackle circumstances (3), fulfill patients' needs, and improve not just the patients' but also their own well-being (4).

Bearing such significant responsibility and engaging compassionately is surely rewarding but exhausting as well (5). It comes with certain consequences of which, one is compassion fatigue. However, it is not only caused by the prior but also from regular and prolonged encounters with clients (6) and situations in work where nurses feel helpless (7). The fundamental urgency 'to do something' (8) and to eliminate others' suffering contributes to the distress, enabling self-dehumanizing tendencies, which commonly strain one's relationship with close ones.

Another effect faced by these nurses is burnout, which descends from compassion fatigue (9), but it occurs when a person is unable to achieve his/ her targets causing frustration, loss of control (10) and impacting patient safety, clinicians' health and workforce retention (9). The top two reasons for compassion fatigue are less administrative support and lack of satisfaction with colleagues (11). Conversely, compassion is a virtuous cycle that though extends from assistance of superiors to care delivery of patients in healthcare but plays a major role specifically in nursing (12). In order to avoid compromising on patient experience, nurses should be trained to be compassion literates (5) by focusing on internal motivation, nurturing self-compassion, increasing staff engagement, and promoting compassionate culture and leadership (13). However, a few programs (e.g. precepting and internships) that have similar aims, have unfortunately been ineffective due to certain disadvantages (14); time and administrative constraints, occasional absences of preceptors, interrupted continuity, discrepancies, and no statistical evidence for cost effectiveness (15). Leadership, precisely on-job mentorship, is considered as a means for generating sustainable benefits in the field of nursing (16).

Mentoring is an interpersonal phenomenon (17) that aims to nurture the well-being of the novice through both informal and formal encounters $(18,19,20)$. Such encounters can be in the form of supporting, guiding, teaching, role modeling, counseling, sharing (21). Even though the formal nursing mentorship 
programs started in the late 1970s (20); however, there has been a lack of focus on building nursing mentorship models (18) and insufficient documentation has been done on mentorship environments present for them (14). Organizing mentorship programs is crucial as they improve the retention rates, enhance job satisfaction $(22,23)$ and reduce compassion fatigue $(24)$. Moreover, confidence, competence, and growth also improve due to the mentoring relationship (25).

It is not just the nurses but patients who have also advocated the need for a support system to sustain compassion in the nursing practice (26). The managers have been identified to be well-placed in this role of mentors given the nature of their relationship with frontline staff. Embedding compassion in the system to be adopted by nurse managers as mentors can be a promising undertaking to teach nurses compassion or as cogently coined by Burridge et al. (2017), 'compassion literacy' (27). A compassionate nursing leader plays a critical role here in filling the void and creating a compassionate culture for nurses, who experience compassion vacuum on the job.

Since, little has been written on how to develop, implement, and sustain nursing compassion for improved patient experience outcomes, this article specifically aims to propose mentoring as an efficient technique to develop compassion focused approaches in on-job nurses. However, it is not just this, but the study also highlights mentoring being a type of micromanagement that can empower nursing staff people in low- and middle - income countries (LMICs). The aim of the present study is to describe the experience of development of a mentorship programme designed to provide on-job compassionate experience to frontline nursing at a systemic level.

\section{Methodology \\ 2.1 Design}

The Theory of Change (ToC) model was utilized to develop a solution for the disengagement among nurses, facilitate relationship building and impart soft skills for instilling a satisfactory employee experience for subsequent satisfactory patient experience. ToC has been widely used and it is considered to be the basis of monitoring and (28) theory-based evaluations $(29,30,31,32)$. According to Weiss (1998), ToC is, "a theory of how and why an initiative works" (33). A robust ToC embodies several elements that makes the model more systematic (28).

The nursing mentorship ToC comprised preconditions, interventions, outcomes and the final goal. The final impact was to achieve improved compassionate experience of patients through refined skills of compassion in nurses. The preconditions are a necessary requirement, condition or element that should be acknowledged for achieving the desired outcome. While in order to fulfill these preconditions or remove dodges, it is important to have efficient interventions that create a positive difference in outcomes and impact of interest (28).

\subsection{Setting}


Established in 1985, Aga Khan University Hospital (AKUH, K) is located in Karachi, the largest city of Pakistan and serves two provinces, Sindh and Balochistan, with populations of 47.89 million and 12.34 million, respectively. It is a tertiary care hospital with consultant doctors specialized to provide optimal care and treatment to patients with a full range and severity of specialty diseases and conditions. Recently, Patient Experience is one of the six approaches among others, including Community and Family Health and Patient Safety and Quality adopted by AKUH, K to facilitate collaborative operations where physicians, nurses, researchers, and teachers work in unison to give patients the best access to care. $\mathrm{AKUH}, \mathrm{K}$ is also Joint Commission International accredited which focuses on patient safety. Through its advanced resources, trained faculty, and technological aid, the institute is creating models for projects rendering short and long-term impact in the provinces. Paediatric service is the largest service line of the hospital having 122 inpatient beds and has the largest number of the nursing services employees $(\mathrm{n}=$ 413) providing high quality, multi-specialty care. All major medical and surgical specialties are present to deliver excellent pediatric care for complex health problems. This nursing management team consisted of a nurse manager as the senior position followed by a specialist and then head nurse and frontline nursing staff. The study was approved for an exemption as Quality Improvement (QI) study by the Ethics Review Committee of the Aga Khan University and as per institutional requirements consent was waived being an exemption.

\subsection{Development of the ToC model}

The development of the model took several steps as described below (Table 1). 
Table 1

The development of ToC Model (Page 8)

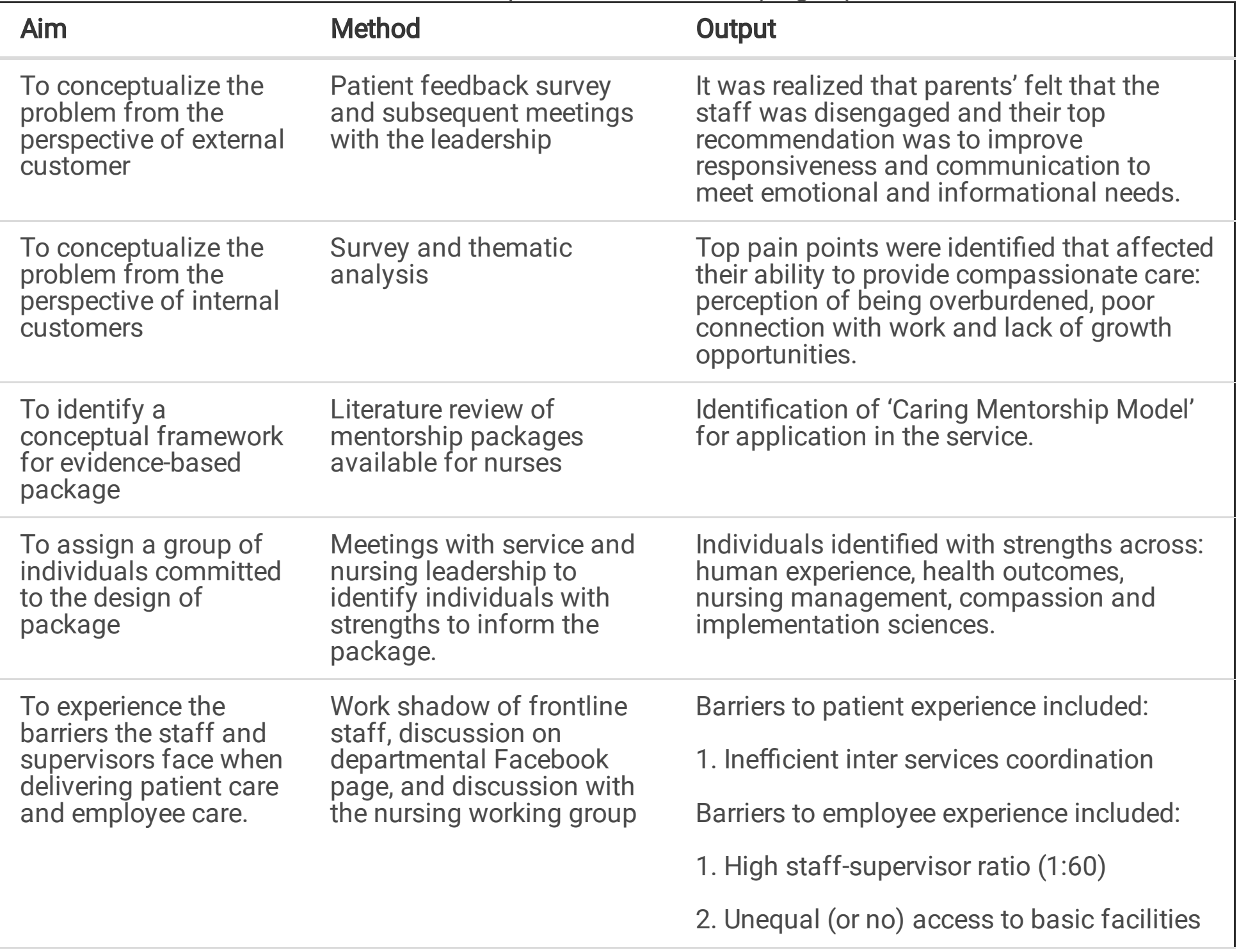




\begin{tabular}{|lll|}
\hline Aim & Method & Output \\
\hline $\begin{array}{l}\text { To identify } \\
\text { preconditions and } \\
\text { design interventions } \\
\text { accordingly. }\end{array}$ & $\begin{array}{l}\text { Regular working group } \\
\text { meetings }\end{array}$ & $\begin{array}{l}\text { The following overarching pre-conditions } \\
\text { were identified: }\end{array}$ \\
& $\begin{array}{l}\text { 1. Hire staff with the right skills for pediatric } \\
\text { care }\end{array}$ \\
2. Define compassionate experience \\
3. Have competent head nurses as \\
supervisors \\
$\begin{array}{l}\text { 4. Create a culture of compassionate } \\
\text { experience }\end{array}$ \\
$\begin{array}{l}\text { The following interventions were agreed on: } \\
\text { 1. Inclusion of paediatric compassion } \\
\text { specific nursing education service (NES) } \\
\text { orientation program } \\
\text { 2. Communication about compassionate } \\
\text { skills, revise career ladder and provide } \\
\text { trainings and on-job coaching } \\
\text { 3. Conduct workshops create checklists and } \\
\text { develop dashboards } \\
\text { 4. Recognize nurses and promote } \\
\text { compassionate values }\end{array}$ \\
\hline
\end{tabular}

\section{- Conceptualization}

As the service leadership and director of patient experience of care sought to work on improving patient experience, a survey was conducted in the paediatric ward with 221 families to determine the issues faced while receiving healthcare which also included items on care from nurses. The survey findings indicated that $75 \%$ of patients reported that nurses always communicated with courtesy and respect, $71 \%$ said nurses listened to them carefully and $77 \%$ felt nurses explained things in a way they could understand. Also, only $27 \%$ reported getting help from staff when they pressed the call bell. There was also an open-ended item requesting families to list down the top three factors that affected their experience during stay. About $38 \%$ indicated being responded to by nursing staff as the first priority followed by effective communication (29\%) and sitting place for attendants was prioritized by $20 \%$. This led to conceptualization of the problem that the staff was disengaged and an intervention to improve patient experience meant engaging the staff via improving their own experience through a 'compassionate mentorship package'.

\section{- Identification of a Framework}

After a literature review, the core team identified the Caring Mentorship Model (34) as a conceptual framework to address the phenomenon of building relationships through mentorship of nursing staff. 
This model has developed from two other models of the Development of Caring Nurse-Self $(35,36)$. It depicts three phases: relationship with no connection, surface connection, and shared connection with each containing three levels that a person reflects at: cognitive (task-oriented mentoring), affective (interactive mentoring), and transformative (transformative mentoring). The program worked on encouraging supervisors to become attuned to mentorship roles, so that improved employee experience and enhanced patient experience. Table 2 states all the questions addressed in accordance with Wagner's model (34) and the activities incorporated by the nursing mentorship programme in each phase.

Table 2

The Addressed Questions as per Wagner's model and the activities suggested by the mentorship manual.

\section{Phases $\quad$ Addressed Questions and Activities}

Task-oriented Understanding is limited to, 'What is happening?' or 'What are the details?'.

Mentoring

In this phase, the mentorship programme focuses on relationship building, ensuring support to the nurses and working on organizational skills.

Interactive

Mentoring

Transformative

Mentoring
Reflecting questions like, 'What is important here?', 'What am I feeling?' or 'Who am I?' (Wagner, 2005a). The manual emphasizes on communication skills, ensuring attendance, encouraging positive interactions, ethics and completing patient notes.

The mentees reflect on questions that caters self and his/ her mentors. These questions include, 'What relationship do I see?', 'What is the meaning?' or 'What more is possible?'. The framework includes play-based interactions and a focus on technical and coordination skills.

Even though the team was unable to formally evaluate as to where the nursing staff stands in terms of mentorship phases but, the baseline assumption suggests the connection was at the cognitive level with surface connection. There was no human connection and mentorship being limited to only monitoring and completing checklists. Hence, the aim was to create opportunities and provide a safe place for the connection to evolve at an 'affective' and later 'transformative' level. It was important to create a humane culture for nurses to reciprocate a similar habitat for patients.

\section{- Creation of a working group}

Next step was identifying a well-organized team with the right skills that was designated to work on this task. The service leadership identified the working group to lead the change effort. Along with the Service Line Chief and Director Patient Experience, members included Nurse Manager, three Nurse Specialists, and a Research Specialist. While the internal group developed technical solutions, external expertise was needed to solidify the compassion angle. Therefore, the team extended to Charter for Compassion Pakistan (henceforth, CfC Pakistan - a local initiative inspired by the Charter for Compassion International) through shared learning of organizational behavior change and nine skills based compassionate programme, respectively, set in motion a mutually beneficial partnership. The team members from $\mathrm{CfC}$ Pakistan included a project lead and an associate.

\section{- Meetings of the working group}


A regular schedule for meetings was carried out to co-design the package to enhance experience of the nursing staff leading to greater patient experience outcomes. Each meeting held discussions on how to achieve the month's agenda and meeting minutes were recorded by the nursing lead. Certain ground rules were set for these conferences which involved application of an empathetic approach, clarity of roles and responsibilities, valuing inputs from all based on their strengths and focus on outcomes and feasibility.

The tasks of the meeting group for implementation of the interventions specifically were divided according to the RACI matrix (Responsible, Accountable, Consulted and Informed). This matrix proves to be beneficial in organizational context as it allows to identify the roles anticipated for each person and any missing roles for early corrections (37). The explanation of each component is as follows:

1. Responsible: The member under this section had this responsibility of achieving task completion. In the current study, e.g. the tasks of designing supervisory checklists and NES pediatric modules were assigned to NS, a nursing specialist who was performing duties for this branch. She was responsible and owned the whole course of action.

2. Accountable: The nursing administrator, NL was accountable to have the final approving authority for the work around supervisory checklists and designing of NES paediatric modules completed by the respective members. Contrary to the 'responsible' team, the accountable one was answerable for the task.

3. Consulted: The consultants directed patient experience of care, (MR), chief of Service Line (BH) and compassion expert (AZ) provided valuable input on the relevant tasks by engaging in two-way communication. They brought in different perspectives regarding pre-conditions and designing modules.

4. Informed: The higher leadership roles i.e. the CNO, Dean of SONAM, CEO and others belonged to this group. They did not provide any input, rather kept a track of needs, preconditions and assessments and developments of interventions.

Moreover, the team was able to design interventions by adopting a systematic process and following a list of core themes. The first step was understanding the contextual needs and then framing the preconditions followed by assessing its effectiveness and feasibility and finally designing modules plus reviewing it as a team. The team went through every minute operational details and set questions based on the focused areas for the interventions (38) mentioned in Table 3. 
Table 3

Key areas of focus for feasibility of the interventions

\begin{tabular}{|ll|}
\hline Areas of Focus & Questions \\
\hline Acceptability & $\begin{array}{l}\text { What is the core issue leading to suboptimal patient experience? } \\
\text { What can be done to eliminate it and prevent it? }\end{array}$ \\
\hline Demand & $\begin{array}{l}\text { How much demand is likely to exist for compassionate on-job mentorship } \\
\text { programmes? }\end{array}$ \\
\hline Implementation & $\begin{array}{l}\text { Who will execute it? } \\
\text { When will it be appropriate to conduct the intervention and who will follow-up } \\
\text { with it? }\end{array}$ \\
\hline Practicality & $\begin{array}{l}\text { Do we have relevant human and financial resources? } \\
\text { Is the method feasible? }\end{array}$ \\
\hline Integration & $\begin{array}{l}\text { Can the compassionate on-job mentorship programme be applied to the adult- } \\
\text { service line? }\end{array}$ \\
\hline $\begin{array}{l}\text { Does the task align with the respective individual's job description? } \\
\text { Himited-efficacy } \\
\text { testing }\end{array}$ & $\begin{array}{l}\text { Will the mentorship process be able to encourage nurses to demonstrate } \\
\text { compassionate behaviors? }\end{array}$ \\
\hline
\end{tabular}

\section{- Needs Assessment}

Pain Points Survey: A brief survey with 173 on-job paediatric in-patient nurses was conducted to assist the working group in realizing what is absent in the experience. The purpose of this survey was to let the nursing staff define their pain points and customize compassion when those pain points are alleviated. The participants revealed numerous pain points that affected their experience. A thematic analysis done by a behaviour implementation scientist (MR) revealed three main themes: perception of being overburdened, lack of connection with work, and lack of growth opportunities. Perception of being overburdened meant that the nurses viewed their work as being excessive with prolonged procedure, night shifts, call on off days, high expectations or pressure from management, excessive documentation etc. While lack of connection with work holds two main themes: accountability and lack of facilities. Lack of facilities included low salary, breaktime issues, one washroom for thirty staff, no medical benefits for their parents, unavailability of instruments, etc. However, accountability catered different issues that according to the nurses were ethically inappropriate. These involved over criticism from seniors, revenge seeking behaviors of co-workers, work environment, and many more. The last pain point i.e. lack of growth opportunities comprised concerns like insecurity of jobs, no promotion of registered nurses (RNs) and no future for aged staff. As per the answers, 'Perception of being overburdened' was found to be the most common theme among the responses. 
Review of existing documents like NES modules, nursing competency checklists led to the realization that few shortcomings had to be addressed to work on patient-centeredness and achieve the final outcome. The existing supervision system or performance management system aimed to assess nurses every 6 months based on a checklist for hard skills only. There was also no training for head nurses to enhance their supervision skills. Whereas, the NES modules were designed for adults only with no integration of pediatric skills which implied that the newly hired nurses had to be retrained once they joined Pediatric service.

\section{Work Shadow}

After the discovery of pain points faced by the nursing staff, it was necessary to identify the workload of nurses during their shift leading to appropriate strategies being made for increasing satisfaction and boosting effective service. Catering to this purpose, it was important to implement an in-depth direct observation to gain a comprehensive understanding of the current settings. Hence, the research team used 'shadowing', which is a technique that involves a researcher following a member of the organization meticulously, over a length of time (39).

In February 2018, a bed-side nurse, a head nurse (administrative supervisor) and an instructor (clinical supervisor) were shadowed, during his/ her work shift by a research associate. According to the observations, the nurse spent most of her time caring for patients and addressing their complaints which was about 25 percent, while patient shifting took the least time. The nurse instructor spent most of her time on nurses' education planning, which was about $23 \%$ (71 minutes) and the head nurse spent most time in rounds i.e. $36 \%$ (111 minutes). There were many challenges identified in nursing staff, inter service coordination including, difficulties in getting their needs fulfilled like lack of lunch facilitation or unavailability of medicines in pharmacy during night. Furthermore, it was revealed that the staffsupervisor ratio was too high, each supervisor supervised more than 64 nurses. Both the supervisors were looking after the same staff but for different reasons (clinical skills and administration). The suggestions to overcome these issues included promoting equality in terms of providing meals and being treated with respect like other cadres or provision of equal facilities. The mantra was to be vigilant and responsive to the workforce i.e. nurses' needs. Once they feel that they are being taken care of, they will resonate a similar attitude towards patients and bring in the best patient care to the healthcare organizations.

\section{Table 4: Components of Compassion in Supervisory Checklists (Page 22)}




\begin{tabular}{|c|c|}
\hline Skills & Example \\
\hline \multicolumn{2}{|l|}{ Soft Skills } \\
\hline Mindfulness & $\begin{array}{l}\text { Accurate assessment, timely identification and appropriate response to patient's } \\
\text { critical needs. }\end{array}$ \\
\hline Courage & Flexibility to accept various assignments as per need of unit/Service Line \\
\hline Altruism & $\begin{array}{l}\text { Guides new staff in provision of safe nursing care and in compliance of Hospital } \\
\text { policies and procedures. }\end{array}$ \\
\hline Gratitude & $\begin{array}{l}\text { Demonstrates gratitude while interacting with the patient's family, colleagues and } \\
\text { immediate supervisors. }\end{array}$ \\
\hline Integrity & Regularity and punctuality with $>98 \%$ attendance and follows leave policy \\
\hline Empathy & Understanding of patient needs and takes proper interventions accordingly \\
\hline $\begin{array}{l}\text { Self- } \\
\text { Compassion }\end{array}$ & Keeps oneself well-groomed and maintains the uniform code at all times \\
\hline Forgiveness & $\begin{array}{l}\text { Demonstrates forgiveness in his/her behavior towards dealing with patient and } \\
\text { colleagues }\end{array}$ \\
\hline Humility & Keep play based interaction with patients. \\
\hline
\end{tabular}

\section{Results}

The model helped to first identify the desired long-term goals and the members worked back from these to identify all conditions (outcomes) that must be in place for the goal to occur. Later, all of these were mapped out in an outcome framework (Table 5 and Figure 1). Correspondingly, the model for nursing mentorship program was developed incorporating the following:

Table 5: Nursing Mentorship ToC (Page 15) 
Impact: Improved compassionate experience of patients (in progress)

$\mathrm{N}(\%)$ patients rating experience with nursing staff as excellent

Intermediate outcome: Improved skills of compassion in nurses

$\mathrm{N}(\%)$ nurses rated as excellent, good, average and poor

\begin{tabular}{|c|c|c|c|}
\hline Assumptions & Interventions & Indicator & Status \\
\hline \multicolumn{4}{|l|}{ Pre-conditions } \\
\hline $\begin{array}{l}\text { 1. Hiring staff with } \\
\text { the right skills } \\
\text { for pediatric } \\
\text { care }\end{array}$ & $\begin{array}{l}\text { Inclusion of paediatric } \\
\text { compassion specific nursing } \\
\text { education service (NES) } \\
\text { orientation program }\end{array}$ & $\begin{array}{l}\mathrm{N}(\%) \text { nurses trained for } \\
\text { pediatric specific } \\
\text { compassionate skills per } \\
\text { batch }\end{array}$ & Completed \\
\hline
\end{tabular}

2.

Compassionate experience defined

as

2.1 Feeling of emotional connectedness with work

\subsection{Formal and} informal on-job training for compassion

2.3 Fair growth opportunities
Create space to talk about compassionate skills with employees

Attendance rates in the workshops

Attrition rates

Provide trainings

$\mathrm{N}(\%)$ nurses trained annually

Not yet

Provide on-job coaching through formal observation and feedback

$\mathrm{N}(\%)$ nurses observed

Revise career ladder of supervisor to be appraised on mentee outcomes
Not yet

$\mathrm{N}(\%)$ attended a professional growth activity every quarter

Initiated
$\mathrm{N}(\%)$ scholarly outputs every quarter 
1. Competent head

nurses as

supervisors

3.1 Training of supervisors as compassionate mentors

Conduct regular training workshops

$\mathrm{N}(\%)$ supervisor trained annually
Done

$\mathrm{N}(\%)$ checklists

completed

Initiated

Developing dashboards
$\mathrm{N}$ times dashboard accessed on a monthly basis per supervisor
1. A culture of compassionate experience
Recognition in town hall meetings, WhatsApp groups and morning huddles

Recognition of workforce also counted towards performance appraisal.
$\mathrm{N}(\%)$ nursing of total appreciation per month
Initiated
$\mathrm{N}(\%)$ patient rating nurses as excellent

$\mathrm{N}(\%)$ of nurses rated as excellent on soft skills (checklists)
Initiated

Not yet
Promotion of compassionate values on department Facebook page
Engagement

$\mathrm{N}(\%)$ posts from nursing staff per month 


\subsection{Impact}

The analysis of patient feedback forms had shown an inadequate responsiveness to patients' needs, due to less engaged workforce. Hence, the impact of all the interventions was agreed to be an improved compassionate experience of patients, provided by the nurses. The indicator for measuring impact was $\mathrm{N}$ (\%) patients rating experience with nursing staff as excellent as measured by Child Hospital Consumer Assessment of Healthcare Providers and Systems (HCAHPS).

\subsection{Intermediate outcomes}

A better employee experience will ultimately enhance patient experience therefore, hence it was ensured to include mentorship of nurses as a core intervention strategy. The intermediate goal included improvement in compassionate skills of nursing staff as indexed by supervisors' rating $\mathrm{N}$ (\%) nurses on performance (good, average and poor) using the checklists. The interventions will be measured using separate indicators and based on this, necessary changes will be proposed. The success of the NES orientation program will be measured depending on the number (\%) of nurses trained for pediatric specific compassionate skills per batch. In order to ensure widespread compassionate experience in every unit, attendance rates in the workshops, attrition rates were taken into consideration. It is also planned to use a number (\%) of nurses trained annually and observed during formal on-job observation during patient care activities, for the said purpose however, it has not been initiated as of yet. Moreover, the outcomes of supervisors' revised career ladder are measured by reviewing the number (\%) of supervisors who attended a professional growth activity and number (\%) of scholarly outputs every quarter.

After training and providing tools to supervisors, its outcome was planned to be measured by observing the number (\%) of supervisors being trained annually, checklists completed and times the dashboard was accessed by a supervisor on a monthly basis. Moreover, the effects of creating groups on communication platforms to increase social engagement was measured through the number (\%) of total appreciation for nurses from peers and other health care professionals and posts from nursing staff per month. Lastly, the performance appraisals will be provided based on receiving 'excellent' ratings on patient feedback form and soft skills checklist.

\subsection{Pre-conditions}

Several pre-conditions were identified in the paediatric service line to achieve the goal of improved patient experience. These were categorized as:

1. Recruit staff with the right skills to achieve the ultimate pediatric care. According to many employers, the issue arises when the required skills are short in supply $(40,41)$. Since, the patient needs are changing now and again, it is necessary for the workforce to be less rigid and adopt new directions to fulfill the needs through 'broad-based' training and skills (42). 
2. Build a compassionate experience to address employee pain points as defined by the nursing staff on the survey: feeling of emotional connectedness with work, formal and informal on-job training for compassion and fair growth opportunities.

3. Maintain or increase competency of head nurses as supervisors by training them as compassionate mentors and making supervisory tools accessible. Mentoring itself is a convoluted process that requires the supervisors to provide constructive feedback and counselling, reflect and encourage and model good teaching practice (43).

4. Create a culture of compassionate experience through instant communication, stimulation of compassionate principles and acknowledgment of the staff's vigorous efforts in providing adequate care. The team employed multiple platforms to impart them with a sense of meaning and promote organizational citizenship behavior including social media. Association of public social communication with risk and ethical issues (privacy, productivity, training and education) has made organizations and the healthcare industry reluctant to adopt it (44), however, recent evidence states otherwise, especially when work is majorly virtual in the face of pandemic (45).

\subsection{Interventions}

This section explains the interventions suggested for the corresponding preconditions however, few of them have already been implemented while others are either initiated but in progress or not commenced at all.

\section{Nursing Education Services (NES) Orientation Module}

The core team included paediatric specific modules in the NES orientation with an introduction to principles of compassion. This was made possible by collaborating with the NES Department at AKUH that conducted basic orientation for all newly hired nurses, at the time of induction. The focus was on basic theoretical and skill competencies required at entry level for safe care provision to patients. However, a coherent approach was applied where the nurses were not only trained for hard skills pertaining to pediatric population but were encouraged to learn soft skills as well, i.e. involve compassion in these orientation trainings so that it was already a part of the new nurses, even before coming on the floor and serving for patient care. Following the initial roll out, refresher sessions were also planned. The intervention was proposed to lead to two main outcomes; improvement in nurses' knowledge and skills regarding care of pediatric patients and skills and compassionate interactions with the patients and their families.

\section{Experiencing compassion at workplace}

It was also aimed to create a conducive space where trainings are provided and talking about compassionate skills with nurses can be made easy. That happens through three pathways:

\section{- On-job connection}


On-job coaching was provided to nurse mentees at bedside level during daily rounds. The nurse mentors observed nurses at work and provided feedback based on the principles of mentorship. They made sure to be available, listen to them, take responsibility and give credit to their mentees, inspire and build a transparent relationship with them, set priorities, model and delegate, focus on professional development and be confident.

\section{- Growth opportunities}

The career ladder for nurses' growth was revised to address their needs for professional growth, the perception of lack of growth and development opportunities. This implied that promotion and compensation will now be received based on the outcome achieved i.e. prime patient care, nurse's experience and credentialing. The minimum requirements for senior positions such as 'Assistant Manager' and 'Nursing Specialist' were updated to 4-5 and 6-8 years of experience respectively. Moreover, receiving compassion skills training and experience in research and publication along with specific certifications such as 'Basic Life Support', 'Safe Medication Administration', 'Conscious Sedation' etc. were made compulsory. With respect to mentoring, these positions are also required to serve as a role model for other staff, conduct needs analysis of unit nurse instructors and identify staff learning needs, develop and implement appropriate teaching interventions and other duties that ensures cost effective patient care outcome.

\section{- Teaching sessions for frontline nurse (mentees) for compassion skills}

Feng et al. (2020) found that out of 10 items, 2 (child comfort and nurse-parent communication) were consistently and strongly associated with willingness-to-recommend scores (46). Hence, it was important to have it as an integrated routine training. As part of the on-job training, regular one-hour teaching sessions are conducted for the nursing staff by their academic supervisor. The sessions were usually designed for at least five days a week and a monthly planner was developed by 22nd of each month. These sessions were planned for fulfilling nurses' academic needs or building their clinical skills. One session per week was designated for compassionate skills to be discussed each week. The presentations and materials were already prepared by the head nurses through an extensive exercise with support from CfC. Few sessions were decided to be conducted by the Physician group while others will be managed by clinical nurse instructor (CNI) and supervised by a Specialist. However, this step could not be implemented in the current situation of Covid-19 pandemic. The nursing staff assignments were modified to meet the need of emerging health emergencies in the hospital. Therefore, the plan to roll out the mentorship checklist will be followed once the current situation is under control.

\section{Strengthened Supervision System}

\section{- Trainings of senior nursing team (managers and specialists)}

A training workshop was planned and conducted by $\mathrm{CiH}$ lead from CfC Pakistan, for the senior nursing team and nurse mentors during June and July 2018 to create opportunities for self-reflection by 
cultivating an understanding of the principles of compassion, namely: self-compassion, mindfulness, empathy, gratitude, forgiveness, courage, integrity, and altruism. This training workshop was created by CfC Pakistan after several visits in all the wards where discussions about the challenges and adversities took place with the on-call nurses and doctors. Supplementing the training workshop on soft skills, i.e. compassion and communication, another workshop was piloted with a focus on hard skills, i.e. the effective supervision tools. These themes revolved around five main abilities; supervision skills, communication skills, observational skills, problem solving and coaching. Following this, a final session was conducted to summarize the overall content through quiz and team discussions. Post training feedback was taken from mentors to ensure they understood the practices that should be implemented for the betterment of nursing experience. A few participants stated in their training evaluation:

"It made me realize to focus on small things in life by showing gratitude, empathy and self-compassion and so on. I think this training will help to ripple the magic of kindness in the whole institution."

"Very engaging and gave a platform for self-reflection."

"Helped us to reflect on ourselves."

\section{- Mentorship of senior managers}

To ensure the success of the mentorship program, another layer of mentorship was included specifically designed for the senior supervisors. The manual created for their supervision established that the specific goals for the nurse mentees should be created in alignment of skills building of the top management and the mentor. An action plan was shaped according to three main dimensions of senior nurse mentor roles and responsibilities: leadership skills, retention talent and transfer of key knowledge, and improving and strengthening culture. Other responsibilities included measuring and categorizing reflections on a scale of absolute to independent reasoning, ensuring that the reflections are progressing according to the month's plan, evaluating head nurses on the basis of competency or value, assessing the skills practices in a day, respecting nurse mentees' personal details and vulnerabilities, finding solutions to eradicate the stress points, and sharing their views about what they think of their mentees. The reflections were not limited to work life; they aimed to gather variables from both personal and professional lives to assess correlations that create both meaningful and toxic results.

\section{- Training of supervisors (head nurses)}

The head nurses also attended a similar 5-day workshop as the senior managers. In the months to follow, special attention was dedicated to preparing the nurse mentors for training of their mentees. The Master Trainer and $\mathrm{CiH}$ lead from CfC Pakistan instated personal visits with each pairing of nurses and advised them on training styles and content. Mock presentations were then scheduled for meticulous feedback to strengthen the delivery of skills to the floor nurses. The supervisor trainings will result in improved skills of the staff to lead compassion trainings for the nursing staff. The outcomes of the training would be a number of staff certified by $\mathrm{CfC}$ to deliver trainings for compassion skills. 


\section{- Supervisory Tools}

A manual was developed for the supervisors (head nurses) to provide an overview of the pediatric nursing mentorship process with all techniques and strategies for supervisors to mentor the floor nurses and standardize the process. The manual also outlined the two modes of mentorship communication; individual and group mentorship. The step-by-step guide suggested forming a flexible work plan with the seniors' consent and reviewing previous records. The manual was designed to respond to such improvements by providing practical situations and steps on how to apply mentorship in the most sustainable way.

\section{- Observing and measuring compassion}

As a part of implementing individual mentorship, it was envisioned that the nurses will be observed (sometimes unannounced as well), which will be followed by a discussion, constructive feedback, and a collaborative discussion on areas to strengthen (up to 3 ) and the ways to do it. Nurses will be assessed by their mentors, using a supervisory checklist which incorporated all the components related to their performance (Table 4). The checklist contained various elements that were objectified around the nine skills of compassion: for instance, nurses' duties, such as regularity and punctuality were counted under 'Integrity', in the checklist. Each nurse was rated on a three-point Likert scale: with 0 being 'no skill' to 3 being 'skill performance with excellency'. Following this, the total score for each section was calculated and the nurse's performance was allotted an overall rate. The objective was to observe every nurse, once a month. After collecting performance data, it will be analyzed on a monthly basis and compared on various aspects: improvement in monthly performances of one mentee, comparative performance of mentees working under one mentor, and performance by different skills. Subsequently, a component named, 'effective stress management' was added in the checklist, to account for psychological health needs of the mentee.

\section{- Data visualization}

An electronic dashboard for supervisors was created to track each frontline nurse's progress, making visual tracking easier (Image 1). Observations were recorded using a tablet and data were synchronized to be displayed in the form of a nursing mentorship dashboard. Everything was monitored in real-time. It also helped to analyze trends in performance and every nurse was color coded as red (overall performance score $\leq 39 \%$ ), yellow (overall score $40-79 \%$ ), or green (overall score $\geq 80 \%$ ). The dashboard was helpful for mentors to compare performance of his/her mentees and was also used to track their growth over a period of time (Image 2). Moreover, it also proved to be convenient to track mentors' performance which further helped the Nursing Manager and Nurse Specialists to monitor performance in a real-time manner. A pilot was conducted in November 2019, at one of the units to examine the feasibility of the approach for receiving bi-monthly feedback to the mentees by their mentors for feedback regarding mentorship checklist from both groups and identify technical glitches while using electronic dashboard for monitoring performance data. Thirty nurses and one head nurse participated in the pilot phase. Majority of the findings revolved around the need to simplify the electronic dashboard 
and data entry mechanism to make it more user-friendly. We had also received opinion on elaborating few components of the mentorship checklist to gain more user clarity. The mentees and mentors had shared a very positive response with the initiation of the compassionate mentorship process.

\section{- Mentorship of supervisors}

A checklist was designed to observe and rate the performance of the supervisors. The supervisors were observed on the basis of five skills with each comprising different responsibilities upon which the mentors were rated; organizational (e.g. punctuality and regularity), technical (observes the staff and provides on job coaching effectively), education (maintains pace during sessions and encourages participation), communication (encourages, praises the staff and also maintains privacy of their performance), and coordination (teamwork and positive attitude). The checklist consisted of a similar three-point Likert scale: 0 being 'no skill' to 3 being 'performance with excellency'. The skills were rated, and a total score was calculated by adding all the ratings.

\section{- Supervisor-nurse ratio}

The new organogram (Figure 2) was more organized with a supervisor-nurse ratio decreased to 1:6. Each unit had its own nursing staff and supervisor to report to. The nursing staff including registrar nurse, registrar midwife, healthcare assistant, patient care attendant, critical care tech and ward assistant, reported to 'Assistant Manager', who further reported to the 'Associate Manager' of the respective unit. Moreover, the new model implied that one supervisor observed the assigned mentees on all skills (i.e. academic and administrative) while practicing compassionate mentorship. This helped the mentee as he/ she only had to refer to one mentor for help and the mentor will be responsible for his/ her growth. Mentors will also be given supervisory checklists to gain a structured feedback mechanism.

\section{Promoting a culture of compassion}

The call for sustainability and continued engagement resulted in another intervention for the nurse mentors; social media engagement. Numerous communication forums were utilized as pathways to create a culture of compassionate experience. This included recognition in town hall meetings, WhatsApp groups, morning huddles and a closed Facebook group. Moreover, appraisals were also planned as another approach to recognize the workforce performance. The nurse appraisal serves several purposes such as determining professional competence, enhancing staff development and motivating them toward higher achievement (47).

\section{- WhatsApp Group}

WhatsApp is known to be a famous application for smartphones that allows both one-to-one and group communication and is often used as a replacement to text messaging (48). The WhatsApp accounts are based on an individual's cell phone number and automatically generates the list of contacts (48). A WhatsApp group named 'Nursing Mentorship Group' was created to promote engagement among mentors through communication, coordination, feedback, and responsiveness. It was envisioned that 
when the Head Nurses are emotionally engaged through this continual knowledge sharing in the form of research, engaging content, real time examples of nurse-patient and mentor-mentee interaction and insightful discussions on the elements of compassion, they will successfully lead compassionate mentorship programme with the 413 nurses of the hospital. Social psychologists predict a link between social group and emotional connection, which may lead to cooperation and positive feelings (49). To this end, the group was also an optimistic attempt to promote openness between nurse mentors and thus, strengthen peer relationships that have encompassing benefits for care providers and ultimately service recipients, i.e. the patients.

\section{- Facebook Group}

Compassionate values were further promoted through a closed Facebook group for the department (50). This group was used among the service line team members and also served as one of the platforms to appreciate high performers among the team. The selection of 'high performers' was based on the appreciation received from patients on the institutional feedback form. Every member shared various informative messages regarding numerous topics that connected them to the organization and its patients for example, newsletters, insights from recent articles and useful tips that can be applied professionally etc. Moreover, achievements of units were also shared. This included the initiative named 'Person of the Week', where the employee was selected on the basis of nomination. Anyone on the Facebook group could suggest a nomination with the page administration team under a criterion shared on the group which included good performance and also showing compassionate behaviors. The name was announced on every Friday with a picture and paragraph written about the person's strength. Out of 82 posts, a total of 18 were dedicated to the nursing team.

\section{Discussion}

This paper presents a mentorship model hinged on compassion as an applied tool for growth and advancement of on-job nurses at a tertiary care hospital in Pakistan. Their unique positioning of caring for patients and collaborating with doctors makes them extremely valuable stakeholders who must be placed at the epicenter of a compassionate healthcare movement. In a system that assigns (almost unapologetically) compassion as a default virtue of all nurses, it was quite a proposition not only to create a programme implementing compassionate mentorship but to predicate that compassion can be taught. It is in this ambition that the programme bears its strength, i.e. sustainability. The programme was postulated on the vision that care attributes begin reflecting in the attitude and actions of the novice nurses when mentors and supervisors become the role model of compassionate values, thus, creating better patient experience. Participant feedback, both from the first set of workshop trainings for mentors and subsequent mentee trainings, concurs that a compassionate mindset can be cultivated by learning about compassion. However, shaping the culture for compassion requires multiple interventions besides training for skills including communication of the value placed on these skills and measurement of the skills. 
The respective paper addresses not only the need of eliminating negative perceptions related to work but also for improvements in the mentoring process. The amalgamation of compassion literacy in the guided framework along with a basic training for mentorship to lead a change can have a positive effect on mentor-mentee relationships through enhanced engagement which ultimately improves patient experience in the healthcare organizations. The study emphasizes immense importance on the role of managers as mentors as they hold a special and unique position in the program. It is through them that sustainability can be achieved along with growth and advancement of on-job nurses, though the outcome is not immediate rather it will be witnessed over the next couple of years. Hence, training should be provided for them to align with their supervisory roles by being objective, concise, skill driven and utilize systemic analyses to overcome the ongoing nursing practice challenges with their mentee team.

CfC Pakistan's Compassionate Skills programme afforded the much-needed objective to form compassion - a deceptively simple concept, often disregarded for difficult measurability and skill transference. The skills were broken down into nine different but interconnected elements, combined with the Master Trainer's expansive repertoire of engagement activities and exercises, invoked relativity and emotionality in the participants who later wrote testimonials for the notable impact on their self-views, both as carers and supervisors. The discourse on compassion was strengthened and reinforced complimenting the changing culture at children's hospital with subsequent visits by the $\mathrm{CiH}$ lead who prepared nurse mentors for the next rollout of trainings. The partnership also yielded the opportunity to engage nurses (rather informally) on WhatsApp wherein a compassionate skill was discussed each week with an emphasis on lived work experiences around it. Expression of gratitude and acknowledgment of colleagues improved on this informal forum.

The collaboration held merit because it favored a shared learning experience for stakeholders at both organizations. A solid evidence for the efficacy of this partnership is the Nurse Performance Checklist technical evaluation protocols for nurses fused with elements of compassion. Success of the programme is also attributed to the well-informed progression planning by the Theory of Change team that created a guided step-by-step framework. Improved wellness, emotional security, interpersonal and intrapersonal connectedness, engagement, and eventually better patient experience are few of the many encouraging outcomes of compassionate mentorship, serving the vision of both partners, i.e. compassionate healthcare. An overwhelming caseload on the nurses and encountering massive deaths, due to the prevalence of global pandemic have caused a decline in mental well-being. Moreover, according to a global study administered by Qualtrics and SAP, almost $40 \%$ of employees from more than ten industries, complained that their companies have not asked 'How they are doing?' since the pandemic (51). Such instances demand for instigating a compassionate framework in organizations to sustain workers' mental health. When asked to indicate their preferences for sharing mental health concerns with, the participants chose peers and managers (38). Hence, a recommendation for future research could be to focus on the application of mentorship guide in other organizations.

A prototype of compassion with provision of equal opportunities and facilities, also aimed to overcome the pain points that were discovered in the thematic analysis. In comparison, the majority of nurses had 
issues of being overburdened due to workload, instead of attaining a lower salary. Hartzband and Groopman indicate a similar circumstance and further reveal that monetary incentives are not a solution for burnout (52). In fact, according to a recent survey, half of the doctors including millennials who have the lowest salaries, were willing to give up minimum $\$ 20,000$ of their annual incomes, in exchange for reduced working hours (53). Burnout can be eliminated from the caregivers' routine through incorporating three main pillars: autonomy, competence and relatedness, in the healthcare profession (54). The current study at some level, utilizes these pillars in the on-job mentorship program to promote a conducive mentor-mentee relation, however its primary focus is to apply a compassionate framework to reduce burnout and compassion fatigue. Absence of Standard Operating Procedures for partnership was a potential limitation in the study. While learnings were shared, and collaboration transpired consultatively, it may be important to develop SOPs as partnership deepens and more actors are involved to achieve the set vision. Lack of planned activities over time, including refresher trainings for the nurse mentors due to their prolonged on-job hours, could also be a potential limitation. However, the likely effects were reconciled through follow-up preparatory sessions and mock presentations with the master trainer. WhatsApp group also served to reinforce learnings from the workshops.

\section{Conclusion}

Nursing communication is known to be a primary driver of family experience in pediatric healthcare. Enhancing their abilities by imparting a compassionate experience using on-job mentorship, enables them to reciprocate compassionate care to their patients. The current study identifies and trains supervisors to provide compassionate experience to nursing staff as a strategy to deal with their pain points at a systemic level in a tertiary care center in a low-to middle -income country. The goal of sustainability can be achieved by aligning mentorship responsibilities as a part of job description and institutional performance management system, when designing mentorship programmes.

\section{Abbreviations}

AKU, K - Aga Khan University (Karachi), ToC - Theory of Change, CfC - Charter for Compassion, RACl Responsible, Accountable, Consulted and Informed, NES - Nursing Education Services, RNs - Registered Nurses, HCAHPS - Hospital Consumer Assessment of Healthcare Providers and Systems, CNI - Clinical Nurse Instructor, $\mathrm{CiH}$ - Compassion in Healthcare.

\section{Declarations}

\section{Ethics approval and consent to participate}

Being a quality improvement project focused on improving patient-centered care, the study was approved as an exemption (2020-5105-11686) by the Aga Khan University Ethics Research Committee. As part of the institutional regulations (AKU ERC), an exemption does not require consent for participation. 


\section{Consent for publication}

Consent was sought from the Ethical Research Committee of the Aga Khan University (AKU) for dissemination of findings.

\section{Availability of data and materials}

The datasets used and/or analyzed during the current study are available from the corresponding author on reasonable request.

\section{Competing interests}

The authors declare that they have no competing interests.

\section{Funding}

No funding was obtained for this study.

\section{Authors' contributions}

AA drafted the original manuscript and led the writing. NL and NS designed interventions including pediatric specific modules, training for the nurse supervisors and the checklists, led the implementation of the interventions and reviewed the final draft. AZ designed and implemented the compassion modules of the trainings. AH designed WhatsApp ToRs and contributed toward writing. $\mathrm{BH}$ conceptualized the idea with MR, contributed towards design of the programme, provided supervision in his capacity as service line chief and reviewed the manuscript drafts. MR conceptualized the study with $\mathrm{BH}$ in her role as director patient experience of care, designed the needs assessment, the mentorship manual, social media strategy, conducted formal analysis, designed the manuscript and contributed toward writing of the drafts. All authors read and approved the final manuscript.

\section{Acknowledgements}

We are thankful to the nursing team for their dedication to the project. Special thanks to the nursing and hospital leadership for supporting the notion of patient-centeredness through employee-centeredness. We would also like to express gratitude to Dr. Abdul Momin Kazi for his support with creation of the dashboards.

\section{References}

1. Frampton SB, Guastello S, Lepore M. Compassion as the foundation of patient-centered care: the importance of compassion in action. Journal of comparative effectiveness research. 2013 Sep; 2(5): 443-55.

2. Dempsey C, Reilly B, Buhlman N. Improving the patient experience: real-world strategies for engaging nurses. JONA: The Journal of Nursing Administration. 2014 Mar 1; 44(3): 142-51. 
3. Bloomfield J, Pegram A. Care, compassion and communication. Nursing Standard (2014+). 2015 Feb 18; 29(25): 45.

4. Saha S, Beach MC, Cooper LA. Patient centeredness, cultural competence and healthcare quality. Journal of the National Medical Association. 2008 Nov 1; 100(11): 1275-85.

5. Burridge L, Winch S, Kay M, Henderson A. What practice nurses need to know about compassion literacy, and why it matters. 2015.

6. Coetzee SK, Klopper HC. Compassion fatigue within nursing practice: A concept analysis. Nursing \& health sciences. 2010 Jun; 12(2): 235-43.

7. Sabo BM. Compassion fatigue and nursing work: can we accurately capture the consequences of caring work?. International journal of nursing practice. 2006 Jun; 12(3): 136-42.

8. Irving JA, Dobkin PL, Park J. Cultivating mindfulness in health care professionals: A review of empirical studies of mindfulness-based stress reduction (MBSR). Complementary therapies in clinical practice. 2009 May 1;15(2):61-6.

9. Hegney DG, Craigie M, Hemsworth D, Osseiran-Moisson R, Aoun S, Francis K, Drury V. Compassion satisfaction, compassion fatigue, anxiety, depression and stress in registered nurses in Australia: study 1 results. Journal of nursing management. 2014 May; 22(4): 506-18.

10. Valent P. Diagnosis and treatment of helper stresses, traumas, and illnesses. Treating compassion fatigue. 2002 Jun 28; 1: 17-38.

11. Lynn MR, Redman RW. Faces of the nursing shortage: influences on staff nurses' intentions to leave their positions or nursing. JONA: The Journal of Nursing Administration. 2005 May 1;35(5):264-70.

12. Straughair $\mathrm{C}$. Exploring compassion: implications for contemporary nursing. Part 1. British Journal of Nursing. 2012 Feb 8;21(3):160-4.

13. De Zulueta PC. Developing compassionate leadership in health care: an integrative review. Journal of healthcare leadership. 2016; 8:1.

14. Angelini DJ. Mentoring in the career development of hospital staff nurses: Models and strategies. Journal of Professional Nursing. 1995 Mar 1; 11(2): 89-97.

15. Goldenberg D. Preceptorship: A one-to-one relationship with a triple " $P$ " rating (preceptor, preceptee, patient). In Nursing Forum 1987 Apr (Vol. 23, No. 1, pp. 10-15). Oxford, UK: Blackwell Publishing Ltd.

16. Marie Block L, Claffey C, Korow MK, McCaffrey R. The value of mentorship within nursing organizations. In nursing forum 2005 Oct (Vol. 40, No. 4, pp. 134-140). Malden, USA: Blackwell Publishing Inc.

17. Gilbert L, Rossman K. Gender and the mentoring process. Women mentoring women: New theoretical perspectives and their application. 1990.

18. Andrews M, Wallis M. Mentorship in nursing: a literature review. Journal of advanced nursing. 1999 Jan; 29(1): 201-7.

19. Tourigny $L$, Pulich M. A critical examination of formal and informal mentoring among nurses. The Health Care Manager. 2005 Jan 1; 24(1): 68-76. 
20. Vance C. Leader interview: mentoring: a professional obligation. Creative Nursing. 2002 Jan 1; 8(3): 4-9.

21. Mayeroff M. On caring. New York: HarperCollins Publishers. 1971.

22. Bowles C, Candela L. First job experiences of recent RN graduates: Improving the work environment. JONA: The Journal of Nursing Administration. 2005 Mar 1; 35(3): 130-7.

23. Hurst S, Koplin-Baucum S. Role acquisition, socialization, and retention: unique aspects of a mentoring program. Journal for Nurses in Professional Development. 2003 Jul 1; 19(4): 176-80.

24. Slatten LA, Carson KD, Carson PP. Compassion fatigue and burnout: What managers should know. The Health Care Manager. 2011 Oct 1;30(4):325-33.

25. Hale RL. Mentorship of nurses: An assessment of the first year of licensure. Lamar UniversityBeaumont; 2004.

26. Straughair C. Cultivating compassion in nursing: A grounded theory study to explore the perceptions of individuals who have experienced nursing care as patients. Nurse education in practice. 2019 Feb 1; 35: 98-103.

27. Burridge LH, Winch S, Kay M, Henderson A. Building compassion literacy: Enabling care in primary health care nursing. Collegian. 2017 Feb 1; 24(1): 85-91.

28. Mayne J. Theory of change analysis: Building robust theories of change. Canadian Journal of Program Evaluation. 2017 Dec 4;32(2).

29. Coryn CL, Noakes LA, Westine CD, Schröter DC. A systematic review of theory-driven evaluation practice from 1990 to 2009. American journal of Evaluation. 2011 Jun; 32(2): 199-226.

30. Donaldson SI. Program theory-driven evaluation science: Strategies and applications. Routledge; 2007 May 21.

31. Funnell SC, Rogers PJ. Purposeful program theory: Effective use of theories of change and logic models. John Wiley \& Sons; 2011 Feb 9.

32. Rogers PJ, Weiss CH. Theory-based evaluation: Reflections ten years on: Theory-based evaluation: Past, present, and future. New directions for evaluation. 2007 Jun;2007(114):63-81.

33. Weiss $\mathrm{CH}$. Methods for studying programs and policies. Wiki-Devel. Sugarlabs. Org. 1998.

34. Wagner AL. A caring mentorship model for nursing: Creating the fabric of caring environments. International Journal for Human Caring. 2005 Jun 15; 9(2): 89.

35. Wagner AL. Connecting to nurse-self through reflective poetic story. International Journal of Human Caring. 2000 Mar 1; 4(2): 7-12.

36. Wagner AL. The embodiment of nursing art: Understanding the caring-self in nursing practice through reflective poetry-writing and art-making. Creating connections between nursing care and the creative arts therapy: Expanding the definition of holistic care. 2005: 229-58.

37. Khan PM, Quraishi KA. Impact of RACI on delivery and outcome of software development projects. In 2014 Fourth International Conference on Advanced Computing \& Communication Technologies 2014 Feb 8 (pp. 177-184). IEEE. 
38. Bowen DJ, Kreuter M, Spring B, Cofta-Woerpel L, Linnan L, Weiner D, Bakken S, Kaplan CP, Squiers L, Fabrizio C, Fernandez M. How we design feasibility studies. American journal of preventive medicine. 2009 May 1;36(5):452-7.

39. McDonald S. Studying actions in context: a qualitative shadowing method for organizational research. Qualitative research. 2005 Nov;5(4):455-73.

40. Cappelli P, Rogovsky N. Self-Assessed Skill Needs and Job Performance. 1995.

41. Galagan P. Bridging the skills gap: Part II. Public Manager. 2010 Jul 1; 39(2): 52.

42. Greenaway D. Securing the future of excellent patient care. London: shape of Training. 2013.

43. Geen A. A Practical Guide to Mentoring: Developing Initial Teacher Training and Education in Schools. UWIC; 2002.

44. Cain J. Social media in health care: the case for organizational policy and employee education. American Journal of Health-System Pharmacy. 2011 Jun 1; 68(11): 1036-40.

45. Jones MS, Goley AL, Alexander BE, Keller SB, Caldwell MM, Buse JB. Inpatient transition to virtual care during COVID-19 pandemic. Diabetes Technology \& Therapeutics. 2020 Jun 1;22(6):444-8.

46. Feng JY, Toomey SL, Elliott MN, Zaslavsky AM, Onorato SE, Schuster MA. Factors associated with family experience in pediatric inpatient care. Pediatrics. 2020 Mar 1; 145(3).

47. Tomey AM. Guide to nursing management and leadership. Australian Nursing and Midwifery Journal. 2008 Jun 1; 15(11): 41.

48. Terpstra M. WhatsApp \& privacy. Radboud University Nijmegen, Netherlands. 2013 Jul 2.

49. Ariffin Z, Omar SZ. Usage of Whatsapp in relation to employee engagement in a telecommunication company. Int. J. Acad. Res. Bus. Soc. Sci. 2018 Jan; 8(1): 434-52.

50. Waleed Sana. Communication: Strategic planning to emotionally engage employees using social media. The Aga Khan University Hospital: The Beryl Institute. 2019. Retrieved from:

https://www.theberylinstitute.org/page/CASE02191.

51. Qualtrics. The other COVID-19 crisis: Mental Health. Employee experience. 2020.

52. Hartzband P, Groopman J. Physician burnout, interrupted. New England Journal of Medicine. 2020 May 1.

53. Kane, L. (2020). Medscape National Physician Burnout \& Suicide Report 2020: The Generational Divide. Medscape. 2020 January 15.

54. Gagné M, Deci EL. Self-determination theory and work motivation. Journal of Organizational behavior. 2005 Jun; 26(4): 331-62.

\section{Supplementary Information}

This paper is accompanied by supplementary information.

Additional File 1: Tables for 'Core Team Members', 'Working Team of the Head Nurses for Training Workshops' and 'Aims, Methods used and Key messages or Opportunities of the Training Sessions' 
Checklists of Nurses and Supervisors

Figure for 'Old Organogram of the Children's Hospital Service Line'

Job Description of Nursing Associate Manager' and Nursing Assistant Manager

\section{Figures}

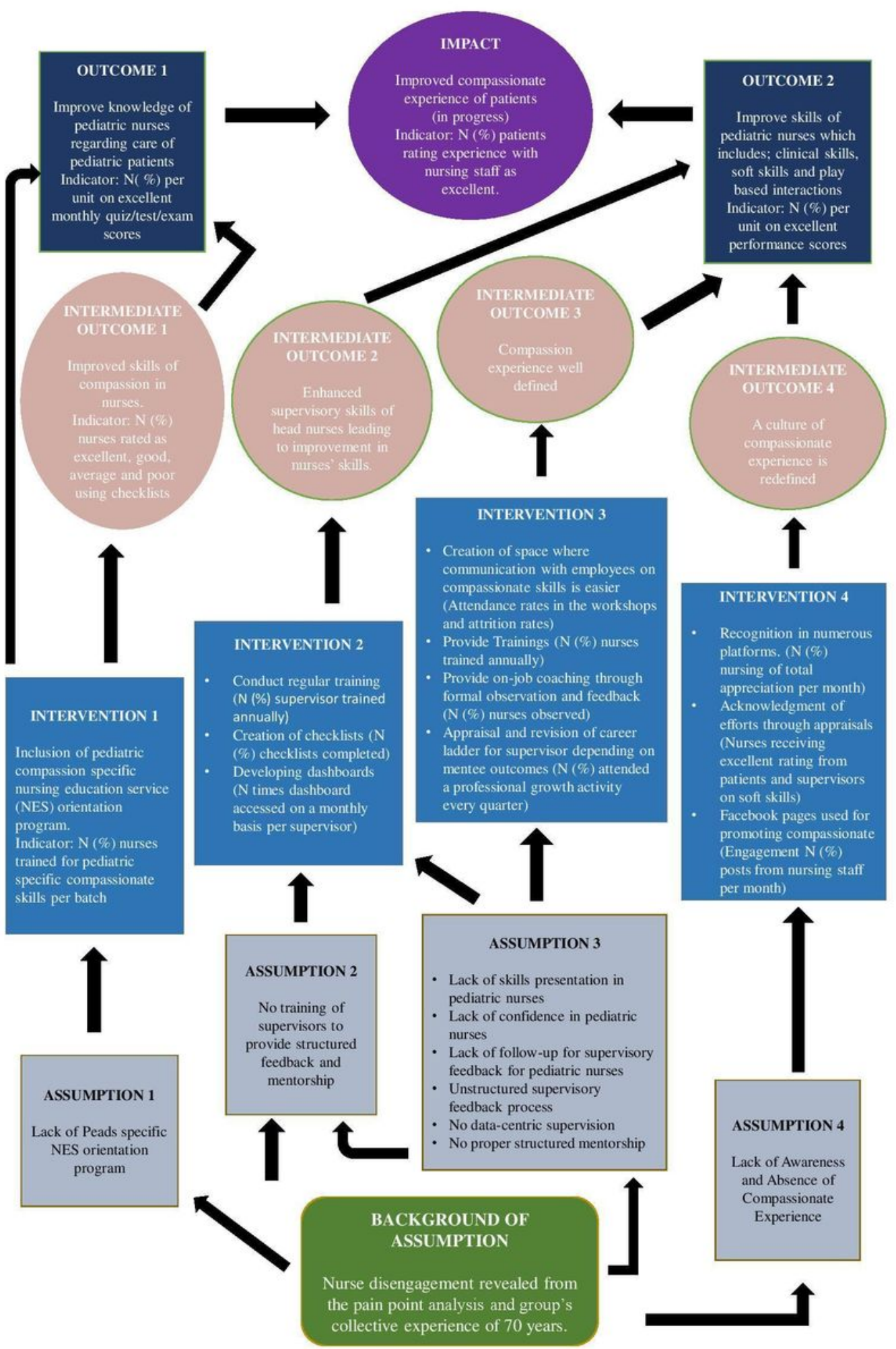


Figure 1

Theory of Change (ToC) formulated by incorporating mentorship and compassion for nurses in children's hospital.

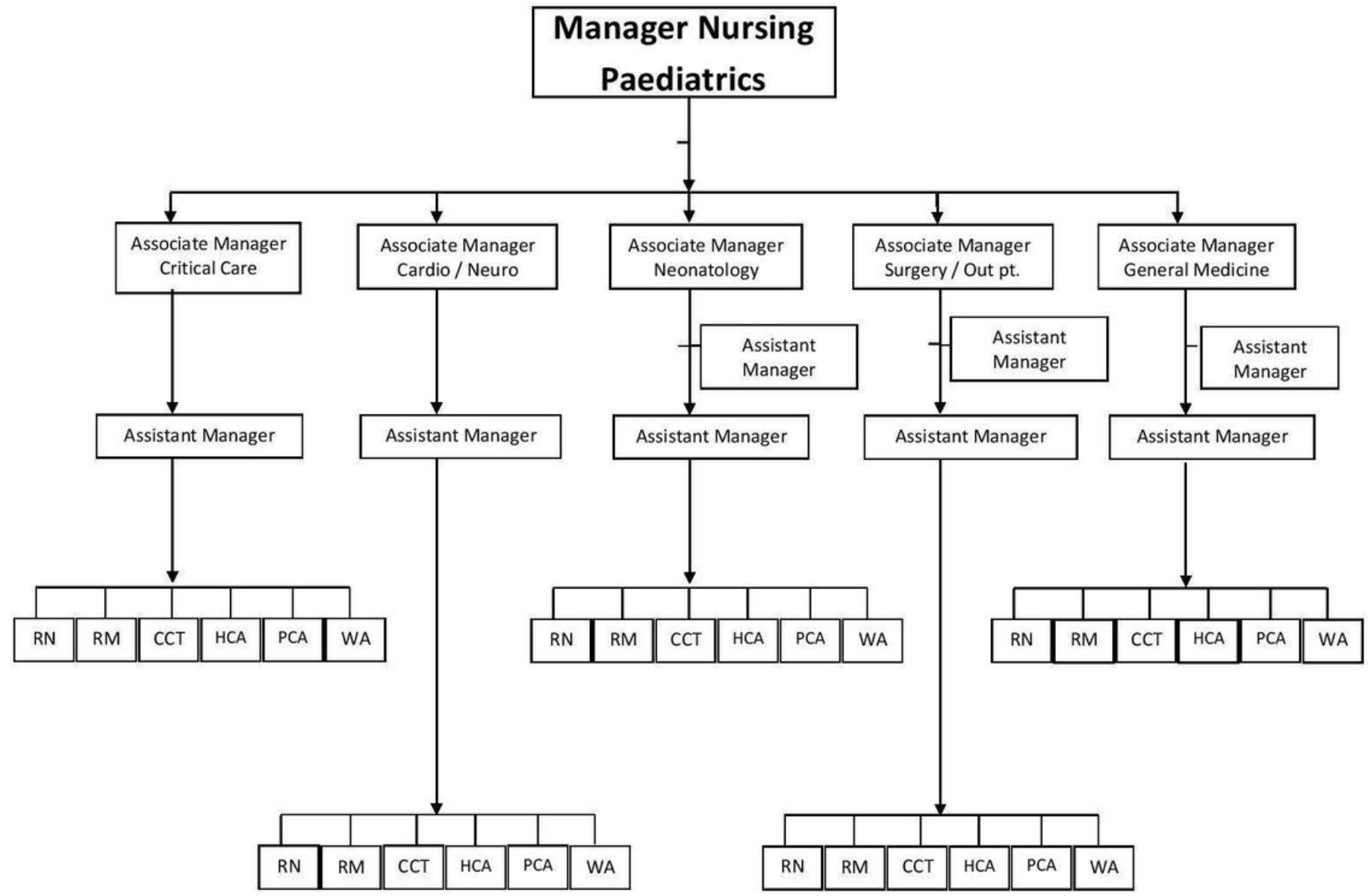

Figure 2

Manager Nursing Paediatrics (The revised organogram) 


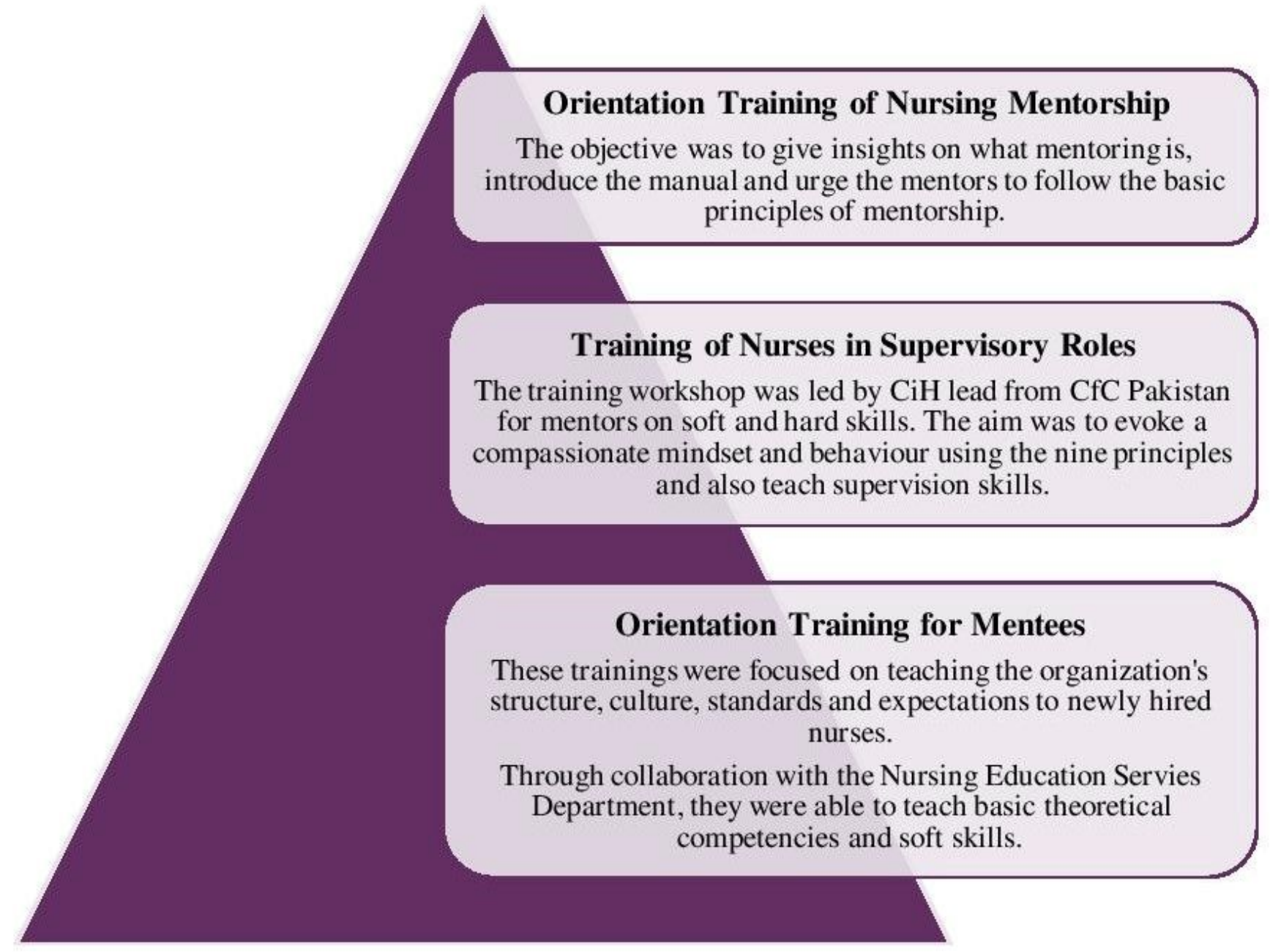

Figure 3

Training conducted for Mentors and Mentees 


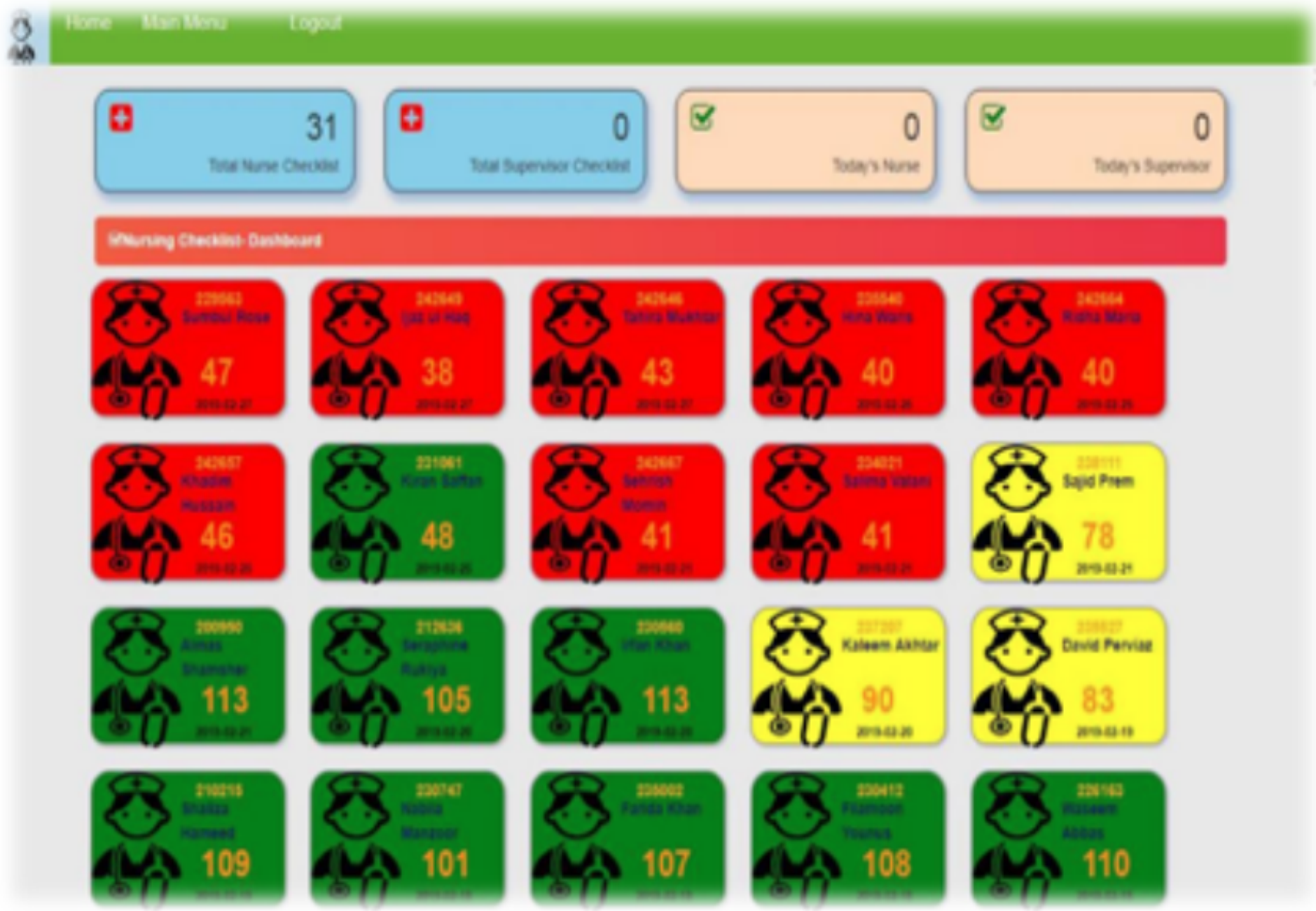

Figure 4

Electronic dashboard used to track each mentee's progress 


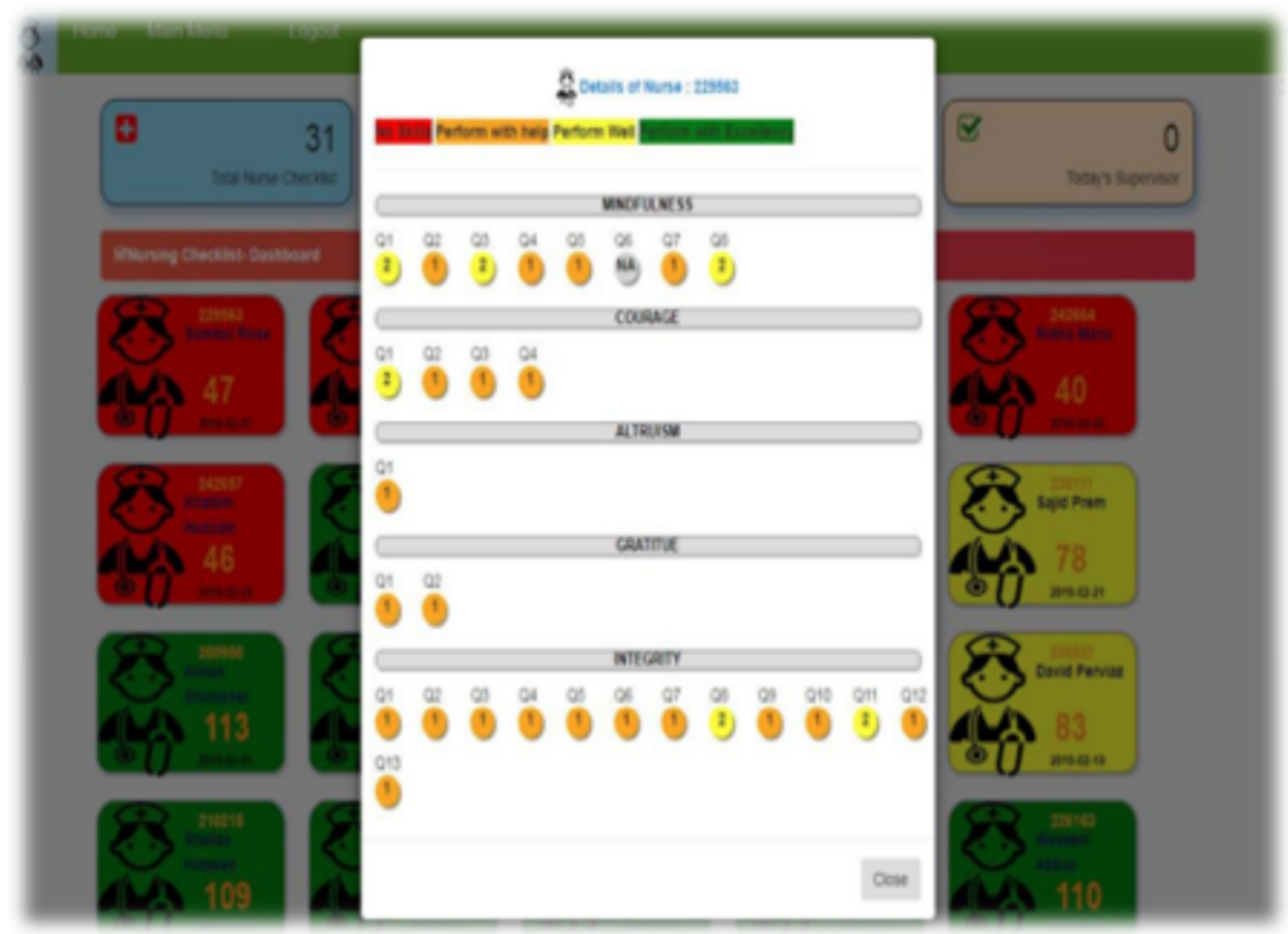

Figure 5

The dashboard tracking growth of the mentee

\section{Supplementary Files}

This is a list of supplementary files associated with this preprint. Click to download.

- AdditionalFile1.pdf 